\title{
¿Es preciso realizar un electrocardiograma en todos los niños con trastorno por déficit de atención e hiperactividad previo al tratamiento con psicoestimulantes?
}

\author{
M.Aparicio Rodrigo, J. González de Dios ${ }^{b}$,J. Ramos Lizana ${ }^{c}$ \\ aPediatra. CS Entrevías. Madrid. España. \\ ${ }^{b}$ Departamento de Pediatría. Hospital General Universitario de Alicante. Alicante. España. \\ 'Servicio de Pediatría. Hospital de Torrecárdenas. Almería. España.
}

Fecha de publicación en Internet: 13 de diciembre de 2011

\begin{abstract}
Resumen
Los autores de los estudios revisados en este artículo no encontraron diferencias en el riesgo de muerte o eventos cardiacos agudos graves entre pacientes con medicación psicoestimulante y sin ella. En conjunto, no consideran adecuada la realización de un electrocardiograma (ECG) o remitir al cardiólogo de forma rutinaria a pacientes con trastorno por déficit de atención con hiperactividad (TDAH) antes de iniciar tratamiento con medicación psicoestimulante. Consideran que la derivación debe basarse en factores de riesgo cardiovasculares detectados a través de la historia clínica o la exploración física.

Comentario de los revisores: con los resultados disponibles no hay evidencia suficiente que justifique la realización de un ECG a todos los niños con TDAH antes del inicio del tratamiento con psicoestimulantes. En estos pacientes, lo prudente sería realizar una historia clínica y una exploración física (incluidas la tensión arterial y la frecuencia cardiaca) y derivar al cardiólogo solo los casos que presenten factores de riesgo cardiovascular o síntomas de enfermedad cardiaca.

Palabras clave: Trastorno por déficit de atención con hiperactividad. Enfermedades cardiovasculares. Muerte súbita. Metilfenidato. Atomoxetina. Estimulantes del sistema nervioso central.

Is it necessary to perform an electrocardiogram in all children with attention deficit disorder before starting treatment with psychostimulants?

Abstract

The authors didn't find differences on the rate of cardiovascular events or death between children exposed and unexposed to stimulant medication. They don't find appropriate to perform an electrocardiogram (EKG) or referral to cardiology of all children with attention deficit and hyperactivity disorder (ADHD) before starting stimulant medication. Cardiology referral should be considered only if cardiac risk factors are detected.
\end{abstract}

María Aparicio Rodrigo, maparicio.gapm01@salud.madrid.org

Este artículo se publica simultáneamente con la revista electrónica Evidencias en Pediatría (www.evidenciasenpediatria.es).

Los autores declaran no presentar conflictos de intereses en relación con la preparación y publicación de este artículo. 
Reviewer's commentary: at present there is not enough evidence to justify the performance of an EKG as screening previous to initiate medical stimulant medication in children with ADHD. It seems reasonable to perform a careful history and physical examination (blood pressure and cardiac frequency included) and referral to cardiology only when cardiac risk factors or cardiac diseases are detected.

Key words: Attention deficit disorder with hyperactivity. Cardiovascular diseases. Cardiac sudden death. Methylphenidate. Atomoxetin. Central nervous system stimulants.

\section{Escenario clínico}

Varón de ocho años, diagnosticado recientemente de trastorno por déficit de atención con hiperactividad (TDAH) por el psiquiatra infantil del centro de Salud Mental. Acude a la consulta de Cardiología Pediátrica de un hospital terciario para realizarle un electrocardiograma (ECG) previo al inicio del tratamiento con psicoestimulantes. Ya es el décimo paciente que se remite a esta consulta por el mismo motivo durante ese mes, cuando previamente no se habían recibido pacientes similares. En todos los casos anteriores se realizó el ECG que se solicitaba y resultó normal. En esta ocasión, el cardiólogo se plantea si esta es una exploración necesaria en todos los niños diagnosticados de TDHA antes de la prescripción de un psicofármaco y decide realizar una búsqueda bibliográfica una vez acabada la consulta.

\section{Pregunta clínica}

En niños diagnosticados de TDAH, ¿es necesario realizar un ECG antes del inicio del tratamiento con psicoestimulantes para prevenir complicaciones cardiovasculares?

\section{Búsqueda bibliográfica}

Fecha de la búsqueda: 28/7/2011

Se efectuó una búsqueda en PubMed y Embase. Se utilizaron los siguientes términos MeSH para confeccionar la pregunta clínica estructurada, con los apartados de paciente ("attention deficit disorder with hiperactivity" or "attention déficit disorder"), intervención ("central nervous system stimulants" or "central stimulant agent" or "adrenérgic uptake inhibitors" or "dextroanphetamine" or "mehylphenidate" or "atomoxetine") y variable de resultado ("cardiovascular diseases" or "heart disease" or "death, sudden"). Se establecieron dos limitaciones en la búsqueda: edad (entre uno y 18 años) e idioma (artículos en inglés y español). También se revisaron las referencias bibliográficas de los artículos encontrados.

Tras la lectura del título y el resumen, se recuperaron aquellos artículos actuales (principalmente de los últimos cinco 
años) que se adaptaran a la pregunta clínica y que tuvieran un diseño de suficiente calidad (estudio de pruebas diagnósticas, ensayo clínico, revisión sistemática, guía de práctica clínica y conferencia de consenso). Se seleccionaron los siguientes estudios ${ }^{1-3}$ :

- Schelleman H, Bilker WB, Strom BL, Kimmel SE, Newcomb C, Guevara JP et al. Cardiovascular events and death in children exposed and unexposed to ADHD Agents. Pediatrics. 2011;127:1102-101.

- Thomas PE, Carlo WF, Decker JA, Cannon BC, Kertesz NJ, Friedman RA et al. Impact of the American Heart Association Scientific Statement on screening electrocardiograms and stimulant medications. Arch Pediatr Adolesc Med. 2011;165:166-70².

- Warren $A E$, Hamilton RM, Bélanger SA, Gray C, Grow RM, Sanatini $S$ et al. Cardiac risk assessment before the use of stimulant medications in children and youth: a joint position statement by the Canadian Paediatric Society, the Canadian Cardiovascular Society and the Canadian Academy of Child and Adolescent Psychiatry. Can J Cardiol. 2009;25: 625-303.
Resumen estructurado de los artículos seleccionados

Schelleman H, Bilker WB, Strom BL, Kimmel SE, Newcomb C, Guevara JP et al. Cardiovascular events and death in children exposed and unexposed to ADHD Agents. Pediatrics. 2011;127: 1102-10.

Objetivo: comparar la tasa de muerte o eventos cardiovasculares graves en niños tratados con psicoestimulantes para el TDAH frente a niños sin este tratamiento.

Diseño: estudio de cohortes retrospectivo.

Emplazamiento: comunitario en Estados Unidos.

Población de estudio: extraída de la base de datos Medicaid (1999-2003) y de la base de investigación "Health core Integrated" de 14 estados (2001-2006). Se seleccionaron todos los niños de edad comprendida entre tres y 17 años en tratamiento con psicoestimulantes. Se diferenciaron dos cohortes expuestas: una cohorte principal de niños con al menos 180 días de observación previos al inicio del tratamiento (cohorte incidente) y otra cohorte secundaria con $<180$ días de observación (cohorte prevalente). Esta diferenciación pretende evitar sesgos de selección a favor de la hipótesis 
nula. La cohorte incidente se componía de 93470 niños tratados con anfetaminas, 128117 con metilfenidato y 19830 con atomoxetina. Cada sujeto del grupo de tratamiento se emparejó con hasta cuatro pacientes sin medicación psicoestimulante en función de la base de datos, tres intervalos de edad, país y sexo $(n=$ $965668)$.

Evaluación del factor de riesgo: se realizó un seguimiento durante una media de 138 días en los pacientes con medicación y de 609 días en los pacientes sin medicación.

Medición de resultado: se consideró como variable principal episodios de hospitalización o asistencia en urgencias por paro cardiaco, arritmia ventricular, infarto de miocardio o accidente cerebrovascular que pudieron ser validados mediante revisión de sus historias clínicas (solo disponibles en el $48 \%$ de los episodios). La validación corrió a cargo de dos cardiólogos pediátricos o dos neurólogos pediátricos y un tercer especialista resolvió las discordancias. Menos del $50 \%$ de los diagnósticos fue validado tras la revisión de las historias. Como variable secundaria, se incluyeron los pacientes fallecidos por cualquier causa, las muertes no debidas a accidentes y las muertes no debidas a suicidio.
Resultados principales: en la cohorte incidente no se encontraron diferencias significativas entre los pacientes con o sin medicación psicoestimulante en la tasa de muerte súbita o arritmia ventricular (hazard ratio o cociente de riesgos instantáneos [CRI]: 1,60, intervalo de confianza del 95\% [IC 95\%]: 0,19 a 13,6) y en todas las causas de muerte (CRI: 0,76, IC $95 \%$ : 0,52 a 1,12); no se identificaron accidentes cerebrovasculares o infartos de miocardio en el grupo tratado con psicomestimulantes. En la cohorte prevalente no se encontraron diferencias significativas entre los grupos de pacientes. Los resultados no variaron cuando se excluyeron a los pacientes con cardiopatías previas.

Conclusión de los autores: los resultados del estudio no sugieren que existan diferencias en el riesgo de muerte o eventos cardiacos agudos graves entre pacientes con medicación psicoestimulante o sin ella.

Conflicto de intereses: algunos autores declaran recibir becas de investigación y/o ser consultores de diferentes compañías (Abbot, Novartis) que fabrican psicoestimulantes.

Fuente de financiación: Shire, compañía farmacéutica americana especializada en tratamiento para el TDAH. 
Thomas PE, Carlo WF, Decker JA, Cannon BC, Kertesz NJ, Friedman RA et al. Impact of the American Heart Association Scientific Statement on screening electrocardiograms and stimulant medications. Arch Pediatr Adolesc Med. 2011;165:166-70.

Objetivo: evaluar la utilidad de la solicitud de un ECG previo al inicio del tratamiento con psicoestimulantes en pacientes con TDAH.

Diseño: estudio descriptivo retrospectivo.

Emplazamiento: hospitalario (hospital terciario en Texas, Estados Unidos).

Población de estudio: pacientes diagnosticados de TDAH derivados al hospital para realizar un ECG antes de comenzar el tratamiento con psicoestimulantes. Se excluyó a los pacientes con enfermedades cardiacas o arritmias diagnosticadas con anterioridad.

Intervención: se comparó el número de ECG realizados, número de ECG patológicos y diagnóstico de enfermedad, en estos últimos, entre el año anterior a las recomendaciones de la American Heart Association (AHA) acerca de realizar un ECG a todos los niños con TDAH antes de iniciar la medicación con psicoestimulantes, y el año posterior.
Medición de resultado: un especialista en electrofisiología revisó todos los ECG. Se analizó el número de ECG patológicos y si se detectó enfermedad en estos pacientes. Como variables secundarias se analizó el número de otras pruebas solicitadas y el cambio en la práctica clínica de los pediatras de Atención Primaria.

Resultados principales: en el periodo previo a las recomendaciones de la AHA se solicitaban una media (M) de 6,9 ECG al mes (desviación estandar [DE]: 3,2), dato que se cuadruplicó tras las recomendaciones de la AHA: M: 31,2 (DE: 9,5). Durante el periodo de estudio se analizaron 372 ECG y se encontraron 24 ECG alterados $(6,4 \%)$. De ellos seis abandonaron el estudio y 18 precisaron más pruebas complementarias, pero en ninguno de los pacientes se detectó enfermedad. No se restringió la medicación psicoestimulante a ningún paciente.

Conclusión de los autores: las recomendaciones de la AHA han conllevado un aumento en la solicitud de ECG y otras pruebas cadiovasculares diagnósticas que conducen a un aumento de costes y un retraso en el inicio del tratamiento. Dado los escasos resultados patológicos de estas pruebas (y hasta que exista mayor evidencia disponible sobre la cardiotoxicidad de los psicoestimulantes) se considera más apropiado 
realizar una historia clínica exhaustiva y exploración física completa y solo solicitar un ECG cuando esté indicado.

Conflicto de intereses: no consta. Fuente de financiación: no consta.

Warren $A E$, Hamilton RM, Bélanger SA, Gray C, Grow RM, Sanatini S et al. Cardiac risk assessment before the use of stimulant medications in children and youth: a joint position statement by the Canadian Paediatric Society, the Canadian Cardiovascular Society and the Canadian Academy of Child and Adolescent Psychiatry. Can J Cardiol. 2009;25:625-30

Objetivo: llegar a un consenso sobre los riesgos de la medicación psicoestimulante.

Fuente de datos: revisión bibliográfica en PubMed (hasta febrero de 2009) y referencias bibliográficas de los artículos encontrados. Revisión de la bibliografía de documentos de consenso previos (AHA y American Academy of Pediatrics [AAP]). Opinión de expertos en muerte súbita y TDAH.

Selección de estudios: se analizaron los títulos y resúmenes de los artículos obtenidos y se seleccionaron los que el grupo de trabajo (miembros de la Sociedad Canadiense de Pediatría, la Sociedad Canadiense de Cardiología y la Sociedad
Canadiense de Psiquiatría del niño y adolescente) consideró oportunos.

Extracción de datos: tres autores realizaron la extracción y el resumen de los datos.

Resultados principales: por el momento, se desconocen los riesgos de la medicación psicoestimulante en pacientes con cardiopatía, aunque se sabe que existe más riesgo de TDAH en niños con cardiopatías estructurales intervenidas. El riesgo de muerte súbita (SMS) en la población infantil general es bajo y varía según los países entre 1,3 y 8,5 por 100 000 habitantes y año, con una media entre 1,2 a 1,3 por 100000 pacientes $y$ año. Con frecuencia, en los casos de SMS existen síntomas o antecedentes familiares que sugieren una situación de riesgo. Las estadísticas muestran que el riesgo de SMS en los niños con TDAH es similar al de la población infantil general. Recomiendan la realización de una historia clínica detallada y una exploración física completa para descartar alteraciones cardiacas (nivel de evidencia C; grado de recomendación Ila), como se haría en cualquier paciente pediátrico, aunque indican que no existe evidencia de su eficacia. El uso del ECG como prueba de cribado se ha evaluado en atletas con resultados variables según los países, pero no hay evidencias de su utilidad 
como cribado en TDAH y, por lo tanto, no hay indicación de hacerlo si la clínica no lo sugiere (nivel de evidencia $C$; grado de recomendación Ila). En pacientes con cardiopatía conocida o arritmias (pacientes que probablemente estén bajo control cardiológico), dado que no existe evidencia de que la medicación en sí aumente el riesgo de SMS, esta se debe prescribir por un especialista en TDAH consultando al cardiólogo, que no tiene por qué ver al paciente (nivel de evidencia $C_{\text {; }}$ grado de recomendación Ila). No hay evidencia que justifique la interconsulta rutinaria con un cardiólogo antes de comenzar el tratamiento con metilfenidato (nivel de evidencia C; grado de recomendación $\mathrm{Ila}$ ), aunque sí se recomienda en pacientes con sospecha o factores de riesgo de enfermedad cardiaca, como se haría en cualquier niño sin TDAH (nivel de evidencia $C_{\text {; }}$ grado de recomendación Ila).

Conclusión: se recomienda hacer una historia clínica detallada y exploración física completa, con especial énfasis en la detección de factores de riesgo para SMS, en pacientes que van a comenzar el tratamiento con psicoestimulantes. No se recomienda la realización de un ECG o la derivación a un cardiólogo de forma rutinaria.

Conflicto de intereses: no consta.

Fuente de financiación: no consta.

\section{Comentario crítico}

Justificación: en el momento actual se calcula que la incidencia de TDHA en escolares es de un 6-10\% (no existen estadísticas fiables en España) y, de ellos, la mayoría reciben tratamiento con psicoestimulantes. Distintos estudios han mostrado que esta medicación puede producir efectos cardiovasculares menores, como un incremento de la presión arterial (de 1-4 $\mathrm{mmHg}$ ) o de la frecuencia cardiaca (de 3-8 latidos por minuto) ${ }^{4}$. La notificación de 25 casos de SMS en pacientes tratados con psicoestimulantes en 2007 despertó la duda sobre su seguridad. Un análisis más detallado de los datos permite concluir que estos 25 casos de SMS notificados a la FDA, considerados en el conjunto de niños que reciben medicación psicoestimulante para el TDAH, suponen un riesgo de SMS similar al de la población infantil general (0,2 a 0,5 por 100000 pacientes y año). Entre los estudios publicados con posterioridad, en los que se confirma este riesgo, destaca el de Gould et al. ${ }^{5}$, que la misma FDA descarta por las deficiencias metodológicas ${ }^{6}$, indicando a los padres que no puede servir como motivo para abandonar la medicación psicoestimulante. Desde entonces se han sucedido diversos estudios que tratan de aclarar este aspecto. 
Validez o rigor científico: los artículos seleccionados, dos de ellos con baja calidad metodológica ${ }^{1,2}$, no aportan suficiente evidencia para descartar el riesgo de SMS en pacientes con TDHA. Aunque, en su conjunto, sugieren que el riesgo (de haberlo) sería mínimo. Hacen falta más estudios para confirmar este hecho, como los tres artículos recomiendan en sus conclusiones.

El primer estudio ${ }^{1}$ fue financiado por la industria farmacéutica. Las cohortes que se comparan presentan importantes diferencias basales: diferencias en contra de los pacientes tratados con psicoestimulantes (que presentaron más antecedentes de enfermedades cardiovasculares y/o factores de riesgo cardiovasculares), lo que habría favorecido a los pacientes no medicados en cuanto a presentar menos muertes y/o eventos cardiovasculares (y no fue así). Solo se pudieron recuperar un $49 \%$ de los casos detectados (aunque este porcentaje fue igual en medicados y no medicados), hecho que afecta de forma importante la validez de los resultados. Es muy escaso el número de eventos validados, lo que implicó que no se pudieran estimar algunos CRI. La incidencia de eventos detectada es inferior a la publicada en otros artículos similares. Los resultados no se pudieron ajustar a variables de confusión, por el escaso número de casos por un lado y el infrarregistro de estas variables en las bases de datos consultadas por otro. Para compensar este aspecto se realizó una comparación de subgrupos con exclusión que, dada la escasez de eventos estudiados, siguió sin dar resultados valorables. Finalmente, no se cuenta con datos de diagnóstico psiquiátrico del TDAH y podrían existir enfermedades cardiacas infradiagnosticadas y no registradas. En conjunto se trata de un estudio con baja calidad metodológica y elevado riesgo de sesgos. La falta de diferencias encontradas no descarta su existencia en la vida real, aunque el análisis de mortalidad (variable no expuesta a sesgos de registro y sin diferencias entre grupos) no sugiere la existencia de un infraregistro diferencial.

El segundo estudio ${ }^{2}$ se fundamenta en un análisis descriptivo de un centro terciario, hecho que afecta la validez externa del estudio. La muestra es escasa y, por tanto, el poder estadístico del estudio es bajo. Se producen pérdidas del $25 \%$ en los pacientes con alteraciones en el ECG, que son excesivas para poder valorar los resultados. Las limitaciones descritas no permiten sacar conclusiones del estudio.

El tercer artículo ${ }^{3}$ es un consenso fundamentado en una revisión sistemática. 
Solo se hace la búsqueda bibliográfica en PubMed (lo que podría haber dejado fuera estudios relevantes publicados en revistas no incluidas en esta base de datos) y no se detalla el método de consenso utilizado, si bien el resto de la metodología es adecuada. El nivel de evidencia de sus recomendaciones es bajo (nivel $C$ : consenso derivado de la opinión de expertos y/o estudios con poca muestra, retrospectivo o basados en registros) y el nivel de evidencia es Ila (evidencias encontradas y/o divergencia en opiniones en relación con la eficacia/utilidad del tratamiento; peso de la evidencia a favor), pero reflejan bien los resultados de los trabajos publicados.

Importancia clínica: los psicoestimulantes ejercen un efecto cardiaco cronotrópico y presor. Es por ello que siempre ha sido de gran interés conocer en pacientes con TDAH (con o sin factores de riesgo cardiovascular) la diferencia en eventos cardiacos adversos, según hayan sido tratados o no con psicoestimulantes. En esta revisión se constata que los tres artículos seleccionados (con diseños diferentes) obtienen conclusiones similares: no existen diferencias en la morbimortalidad cardiovascular en niños con TDAH, por lo que la realización sistemática de ECG antes de iniciar la medicación psicoestimulante no aporta beneficios. Es cierto que existen limitaciones en estos estudios que impiden asegurar la importancia cuantitativa y cualitativa de estos datos (y que se necesitan estudios más adecuados para responder a la cuestión clave del potencial aumento de SMS, para lo cual la FDA está llevando a cabo un estudio epidemiológico con 500000 pacientes ${ }^{6}$ ), pero ello no es óbice para que estas conclusiones también se plasmen en las guías europeas 2011 sobre el manejo de los efectos adversos en la medicación del TDAH7 ${ }^{7}$. La realización sistemática de ECG antes de iniciar el tratamiento con psicoestimulantes en niños con TDAH tiene una relación beneficioriesgo-coste desfavorable. El beneficio consistiría en el potencial hallazgo de alteraciones ECG asociadas a SMS (QT largo, por ejemplo); la prueba en sí no tiene riesgos, pero sí lo tiene el programa de cribado, "riesgos" intrínsecos de todo cribado 8 : aumento de la ansiedad familiar, falsos positivos, o fenómeno de etiquetado. Respecto a los costes, cabe considerar no solo el coste en personal y tiempo para la realización e interpretación del ECG, sino también el manejo de los falsos positivos.

Los estudios actuales contradicen claramente la posición inicial de la AHA en el año $2008^{9}$, que apoyaba la realización de ECG. Aun así, podemos encontrar al- 
gún estudio de evaluación económica en que, tras la modelización, se obtiene que realizar rutinariamente ECG (junto a la historia y la exploración física) a niños con TDAH antes de iniciar el tratamiento con psicoestimulantes, tiene un costeefectividad límite (frente a la práctica clínica habitual) para prevenir el SMS en relación con los años de vida ajustados por calidad ${ }^{10}$. Este modelo tiene dos limitaciones claras: la debilidad de las asunciones (fundamentadas en estudios observacionales y de cohortes) y el no considerar el "coste" derivado de los riesgos intrínsecos de un cribado masivo (fenómeno de etiquetado, ansiedad familiar, falsos positivos, etc.).

Aplicabilidad a la práctica clínica: la incidencia de SMS en pacientes con TDAH que reciben tratamiento con psicoestimulantes entra dentro del amplio rango de lo esperable en población pediátrica, que varía desde un 1,3 a 8,5 por 100000 habitantes y año, según los países. En el caso de existir un riesgo de la medicación psicoestimulante, este sería mínimo y no ha podido descartarse en los estudios disponibles debido a la baja incidencia de SMS en niños, lo que obligaría a disponer de un tamaño muestral mayor para poder demostrar/descartar el efecto. Hasta el momento no existe publicado ningún estudio con estas caracte- rísticas, pero sí hay uno en marcha promovido por la $\mathrm{FDA}^{6}$, del que se están analizando los resultados preliminares. Por el momento (y con los datos disponibles) parece que el riesgo de SMS debido a psicoestimulantes es inexistente o mínimo. Por lo tanto no se justifica, a la luz de los conocimientos actualmente disponibles, realizar en estos pacientes pruebas complementarias de forma sistemática. Hasta que haya más datos definitivos, parece que lo más razonable es descartar, mediante la historia clínica y la exploración física, la existencia de factores de riesgo cardiaco antes de iniciar la medicación. En caso de hallarlos, se deben remitir al cardiólogo para valoración antes de comenzar el tratamiento, que no estaría contraindicado.

El camino del TDAH está sembrado de sobrediagnóstico y sobretratamiento ${ }^{11}$. no lo llenemos de sobrecribado.

\section{Conflicto de intereses de los autores} del comentario: no existe.

\section{Resolución del escenario}

Con los resultados disponibles se concluye que no hay evidencia suficiente que justifique la realización de un ECG a todos los niños con TDAH previo al inicio de la medicación con psicoestimulantes. Con estos resultados, nuestro cardiólogo infantil decidió convocar una sesión (in- 
vitando a los pediatras de Atención Primaria, psiquiatras y psicólogos del centro de Salud Mental) para explicarles los resultados de su búsqueda y marcar unas directrices para el manejo futuro de estos pacientes.

Sobre la base de las recomendaciones actuales, en niños con tratamiento correcto de TDAH y que precisen medicación psicoestimulante, lo prudente es:

- Historia clínica y exploración física para despistaje de enfermedad cardiovascular.
- A estos pacientes se les debe monitorizar la presión arterial y frecuencia cardiaca al inicio y cada tres o seis meses, una vez iniciada la medicación psicoestimulante.

- La derivación al cardiólogo se realizará solo en los que tengan factores de riesgo y/o síntomas de enfermedad cardiaca. Pero no solicitar ECG rutinario al inicio de la medicación psicoestimulante.

\section{Bibliografía}

1. Schelleman $H$, Bilker WB, Strom BL, Kimmel SE, Newcomb C, Guevara JP et al. Cardiovascular events and death in children exposed and unexposed to ADHD Agents. Pediatrics. 2011;127: 1102-10.

2. Thomas PE, Carlo WF, Decker JA, Cannon $B C$, Kertesz NJ, Friedman RA et al. Impact of the American Heart Association Scientific Statement on screening electrocardiograms and stimulant medications. Arch Pediatr Adolesc Med. 2011;165:166-70.

3. Warren $A E$, Hamilton RM, Bélanger SA, Gray C, Grow RM, Sanatini $S$ et al. Cardiac risk assessment before the use of stimulant medications in children and youth: a joint position statement by the Canadian Paediatric Society, the Canadian Cardiovascular Society and the Canadian Academy of Child and Adolescent Psychiatry. Can J Cardiol. 2009;25:625-30.

4. Silva RR, Skimming JW, Muniz R. Cardiovascular safety of stimulant medications for pediatric attention-deficit hyperactivity disorder. Clin Pediatr (Phila). 2010;840-51.

5. Gould MS, Walsh BT, Munfakh JL, Kleinman M, Duan N, Olfson M y cols. Sudden death and use of stimulant medication in youths. Am J Psychiatry 2009;166:992-1001.

6. Communication about an Ongoing Safety Review of Stimulant Medications used in Children with Attention-Deficit/Hyperactivity Disorder (ADHD). [En línea] [fecha de lectura: 10-VIII2011]. Disponible en FDA U.S. Food and Drug Administration.

7. Graham J, Banaschewski T, Buitellar J, Coghill D, Danckaerts M, Dittmann RW et al. European guidelines on managing adverse effects of medication for ADHD. Eur Child Adolesc Psychiatry. 2011;20:17-37.

8. González de Dios J, Mollar Masedes J, Rebagliato Russo M. Evaluación de los programas y pruebas de detección precoz (cribado o screening) de enfermedades. Rev Pediatr Aten Primaria. 2005;7:593-617. 
9. Vetter VL, Elia J, Erickson C, Berger C, Berger $\mathrm{S}$, Blum $\mathrm{N}$ et al. American Heart Association Council on Cardiovascular Disease in the Young Congenital Cardiac Defects Committee; American Heart Association Council on Cardiovascular Nursing. Cardiovascular monitoring of children and adolescents with heart disease receiving medications for attention deficit/hyperactivity disorder [corrected]: a scientific statement from the American Heart Association Council on Cardiovascular Disease in the Young Congenital Cardiac Defects Committee and the Council on Cardiovascular Nursing. Circulation. 2008;117:2407-23.
10. Denchev $P$, Kaltman JR, Schoenbaum $M$ Vitiello B. Modeled evaluation of alternative strategies to reduce sudden cardiac death among children treated for attention deficit/hyperactivity disorder. Circulation. 2010;121:1329-37.

11. González de Dios J, Cardó E, Servera M. Metilfenidato en el trastorno por déficit de atención e hiperactividad (TDAH): ¿realizamos una práctica clínica adecuada? Rev Neurol. 2006;43: 705-14. 